\title{
Selective aldosterone blocker ameliorates the progression of non-alcoholic steatohepatitis in rats
}

\author{
RYUICHI NOGUCHI, HITOSHI YOSHIJI, YASUHIDE IKENAKA, KOSUKE KAJI, YUSAKU SHIRAI, \\ YOSUKE AIHARA, MASAHARU YAMAZAKI, TADASHI NAMISAKI, MITSUTERU KITADE, \\ JUNICHI YOSHII, KOJI YANASE, HIDETO KAWARATANI, TATSUHIRO TSUJIMOTO and HIROSHI FUKUI
}

Third Department of Internal Medicine, Nara Medical University, Nara 634-8522, Japan

Received April 16, 2010; Accepted June 14, 2010

DOI: 10.3892/ijmm_00000480

\begin{abstract}
Although non-alcoholic steatohepatitis (NASH) may progress to cirrhosis and hepatocellular carcinoma (HCC), no effective therapeutic modalities have been fully established yet. Recent studies have shown that the reninangiotensin-aldosterone-system plays an important role in NASH. The aim of our current study was to elucidate the effects of aldosterone (Ald) inhibition on the progression of $\mathrm{NASH}$. In the choline-deficient L-amino acid-defined dietinduced rat NASH model, the effects of a clinically used selective Ald blocker (SAB) were elucidated in conjunction with the activated hepatic stellate cells (HSC) and neovascularization, which are both known to play important roles in liver fibrosis development and hepatocarcinogenesis, respectively. Liver fibrosis development and the glutathione-S-transferase placental form-positive pre-neoplastic lesions were both markedly attenuated by SAB along with the suppression of the activated HSC and neovascularization. SAB inhibited the hepatic expression of transforming growth factor- $\beta 1$ and also that of the vascular endothelial growth factor. Our in vitro study showed that SAB also inhibited the Ald-induced HSC proliferation and in vitro angiogenesis in a dose-dependent manner. These results indicated that Ald plays a pivotal role in the progression of NASH. Considering that SAB is already widely used in clinical practice, this drug could represent a potential new strategy against NASH in the future.
\end{abstract}

Correspondence to: Dr Hitoshi Yoshiji, Third Department of Internal Medicine, Nara Medical University, Shijo-cho 840, Kashihara, Nara 634-8522, Japan

E-mail: yoshijih@naramed-u.ac.jp

Abbreviations: ACE-I, angiotensin-converting enzyme inhibitor; Ald, aldosterone; AT-II, angiotensin-II; EC, endothelial cells; HCC, hepatocellular carcinoma; HSC, hepatic stellate cells; NAFLD, nonalcoholic fatty liver disease; NASH, non-alcoholic steatohepatitis; $\mathrm{SAB}$, selective aldosterone blocker; VEGF, vascular endothelial growth factor

Key words: aldosterone, selective aldosterone blocker, liver fibrosis, hepatocarcinogenesis, angiogenesis

\section{Introduction}

Non-alchoholic fatty liver disease (NAFLD) ranges from simple steatosis to cirrhosis. Fatty liver has been recognized as a benign and non-progressive condition (1). However, non-alcoholic steatohepatitis (NASH) is now widely known as a potent liver disease which may progress to liver cirrhosis and finally hepatocellular carcinoma (HCC) $(2,3)$. Patients with NAFLD have frequently presented clinical complications, such as obesity, type 2 diabetes mellitus, and insulin resistance (4). While sustained weight loss should be very effective in improving NAFLD, it is somewhat difficult for many patients to change their lifestyle. Accordingly, efforts are currently directed worldwide at overcoming NAFLD, especially NASH. However, no widely accepted therapeutic modalities have been established in clinical practice.

The renin-angiotensin-aldosterone system (RAAS) is a key mediator in the regulation of arterial blood pressure and body fluid homeostasis, and plays an important role in the regulation of local hemodynamics in several organs. It has been reported that angiotensin-II (AT-II), an octapeptide produced via the enzymatic cleavage of angiotensin-I by the angiotensin-I converting enzyme (ACE), plays an important role in the progression of chronic liver diseases (5-7). Intrahepatic RAAS is up-regulated in experimental hepatic fibrosis, and the serum AT-II levels are frequently elevated in patients with cirrhosis $(8,9)$. The AT-II antagonists demonstrated antifibrotic activity in the animal models of NASH and in clinical practice $(10,11)$. Aldosterone (Ald), which is positioned downstream of AT-II in RAAS, is a mineralocorticoid hormone that acts classically on blood pressure regulation and the electrolyte balance via the mineralocorticoid receptors (MR). In addition to Ang II, Ald is also implicated in liver damage and fibrosis (12). However, the interaction between Ald and the progression of NASH is still obscure.

In addition to liver fibrosis, AT-II plays a pivotal role in HCC growth and hepatocarcinogenesis $(7,13)$. It is now widely recognized that angiogenesis is a crucial step in the development of tumors, including HCC (14-16). Several studies have revealed that neovascularization and angiogenic factors, such as the vascular endothelial growth factor (VEGF), are significantly up-regulated in human HCC samples (16-18). Therapies targeting the tumor vessels have proven successful for cancer treatment in experimental models 
$(16,19,20)$. Several anti-angiogenic agents have already been employed in clinical practice (21). Sorafenib, a multi-kinase inhibitor that also targets VEGF receptors, has clearly improved (i.e., prolonged) the overall survival of patients with advanced HCC, and this drug is already approved in many countries for the treatment of HCC (22). It is highly probable that sorafenib will become the standard therapeutic agent for the cure of the advanced stages of HCC in the near future, but the long-term administration of this agent to cirrhotic patients could lead to severe side-effects since drug metabolism is usually hypoactive in these patients. As such, almost all patients show adverse reactions with sorafenib, such as hand-foot syndrome, and certain symptoms are very severe. Furthermore, these recently developed agents are very expensive (23). An alternative approach could be to find a clinically available compound that also exhibits anti-angiogenic activity, of which the safety of long-term administration has been proven.

We have previously reported that AT-II suppression by the ACE inhibitor suppressed HCC growth and hepatocarcinogenesis via angiogenesis inhibition $(5,7,16)$. Similar to AT-II, it has been suggested that Ald plays an important role in angiogenesis. Now, a selective Ald blocker (SAB) is clinically used for the treatment of hypertension and heart failure in many countries, including Japan. We found that treatment with SAB exerted a marked inhibitory effect on the HCC growth along with the suppression of VEGF-mediated angiogenesis (24). However, the role of Ald and the effects of SAB on hepatocarcinogenesis have not yet been elucidated.

The present study was conducted in order to examine the effects of SAB on liver fibrosis and hepatocarcinogenesis in a rat NASH model induced by rats feeding on a cholinedeficient, L-amino acid-defined diet (CDAA) $(10,25)$. We also attempted to investigate the possible mechanisms involved.

\section{Materials and methods}

Animals and reagents. A total of 30 male 6-week-old Fischer 344 rats were purchased from Japan SLC, Inc. (Hamamatsu, Shizuoka, Japan). They were housed under controlled temperature conditions and relative humidity, with 10-15 air changes/h and light illumination for $12 \mathrm{~h} /$ day. SAB was purchased from Pfizer Inc. (Tokyo, Japan). Ald and conventional chemical agents were purchased from Nacalai Tesque (Kyoto, Japan). Control solutions were prepared in ethanol for Ald and in dimethyl sulfoxide (DMSO) for SAB. The human umbilical vascular endothelial cells (HUVECs) were obtained from the Japanese Cancer Research Resources Bank (Tokyo, Japan) and Kurabo (Osaka, Japan). The cells were grown in a supplier-recommended medium.

Animal treatment. The experimental period in all experiments was 16 weeks. Group $(\mathrm{G}) 1$ consisted of untreated rats that served as the control group, and received a choline-sufficient L-amino acid-defined (CSAA) diet. The rats in G2 and G3 received CDAA diet. In G3, the rats received $100 \mathrm{mg} / \mathrm{kg} /$ day of SAB starting from week 12 by gavage. The dose of SAB in the current study was almost comparable to that used in clinical practice (26). The animals were allowed free access to food and water throughout the acclimation and experimental protocols. The rats were sacrificed on day 84 (week 12). Several serum markers, such as alanine aminotransferase (ALT), were assessed by routine laboratory methods. All animal procedures were performed according to approved protocols and in accordance with the recommendations for the proper care and use of laboratory animals.

Histological and immunohistochemical staining and quantification. In all the experimental groups, the first section was routinely stained with hematoxylin and eosin for histological examination. Another section was stained with Sirius red for the detection of fibrosis development. Immunohistochemical staining for the $\alpha$-smooth muscle actin ( $\alpha$-SMA) (Dako, Kyoto, Japan) and the enzyme-altered pre-neoplastic lesions; namely, the placental form of glutathione-S-transferase (GST-P) (MBL Co. Ltd., Nagoya, Japan), were performed as described previously (27). For the determination of in vivo angiogenesis, we performed the immunohistochemical analysis of the platelet/EC adhesion molecule CD31, which is widely used as a marker of neovascularization, in frozen sections of the liver as described previously (28). The immunostained microvessel length was assessed under 200x magnification. In counting, the large vessels with a thick muscular wall or with a lumen greater than $50 \mu \mathrm{m}$ in diameter were excluded. These immunopositive vessels were evaluated with Adobe Photoshop and NIH image software as described previously (29).

Expression of transforming growth factor (TGF)- $\beta$ and $V E G F$ in the liver. The TGF- $\beta$ and VEGF expression levels in the liver were measured by an enzyme-linked immunosorbent assay (ELISA) kit (Bender MedSystems and R\&D Systems, Tokyo, Japan) according to the manufacturer's instructions. Liver lysates were prepared as described previously (30), and the samples were equalized for protein concentration prior to measuring the protein levels.

In vitro assays. The hepatic stellate cells (HSC) were purified from F344 rats by the sequential digestion of the liver with pronase and collagenase as previously described (31). The cell purity, assessed by examining the autofluorescence properties of the stored retinoids in HSC, was typically between 90 and 95\%. HSC were cultured in Dulbecco's Modified Eagle's Medium (Nakarai, Kyoto, Japan) supplemented with $10 \%$ fetal bovine serum and $2 \mathrm{mM}$ L-glutamine and then cultured in a $95 \%$ air- $5 \% \mathrm{CO}_{2}$ humidified atmosphere at $37^{\circ} \mathrm{C}$. The growth medium was changed every other day. HSC were activated by culturing on plastic for 7 days (32). The effects of Ald and SAB on the proliferation of HSC and HUVECs were determined by tetrazolium, 3-(4, 5-dimethylthiazol-2,5yl)-2,5-diphenyltetrazolium bromide (MTT) assay as described previously (33). The cell proliferation was quantified via the conversion of MTT by cells cultured in 48well plates. The cells were incubated with Ald and/or SAB at various concentrations for $24 \mathrm{~h}$, and MTT was added to each well at a final concentration of $5 \mathrm{mg} / \mathrm{ml}$. After 4-h incubation at $37^{\circ} \mathrm{C}$ with $\mathrm{MTT}$, the untreated MTT and medium were removed and $2 \mathrm{ml}$ DMSO were added to solubilize the MTT formazan. After gentle agitation for $10 \mathrm{~min}$, the absorbance of each well, which is directly proportional to the number of 
Table I. The experimental group given the CDAA diet with Epl.

\begin{tabular}{lccc}
\hline & $\begin{array}{c}\text { CSAA } \\
(\mathrm{G} 1)\end{array}$ & $\begin{array}{c}\text { CDAA } \\
(\mathrm{G} 2)\end{array}$ & $\begin{array}{c}\text { CDAA+SAB } \\
(\mathrm{G} 3)\end{array}$ \\
\hline Effective no. of rats & 10 & 10 & 10 \\
Final body weight $(\mathrm{g})^{\mathrm{a}}$ & $95 \pm 20$ & $12 \pm 20^{\mathrm{c}}$ & $210 \pm 18^{\mathrm{c}}$ \\
Relative liver weight & $2.7 \pm 0.4$ & $3.2 \pm 0.5^{\mathrm{b}}$ & $3.1 \pm 0.4^{\mathrm{b}}$ \\
(g/100g body wt.) & & & \\
ALT (IU/l) & $54.4 \pm 18.7$ & $275.5 \pm 18.4^{\mathrm{c}}$ & $324.2 \pm 72.1^{\mathrm{c}}$ \\
Alb (g/dl) & $4.0 \pm 0.3$ & $4.2 \pm 0.5$ & $4.0 \pm 0.2$ \\
T-bi (mg/dl) & $0.10 \pm 0.03$ & $0.12 \pm 0.02$ & $0.12 \pm 0.02$ \\
\hline
\end{tabular}

${ }^{a}$ Data represent mean $\pm \mathrm{SD}$. ${ }^{\mathrm{b}, \mathrm{c}}$ Statisticaly significant compared to G1 $\left({ }^{\mathrm{b}} \mathrm{P}<0.05,{ }^{\mathrm{c}}<0.01\right)$.

living cells, was measured with a 540-nm filter. The effects of Ald and $\mathrm{SAB}$ on the extracellular-signal regulated kinase 1/2 (ERK1/2) phosphorylation in HUVECs were evaluated by the Cellular Activation of Signaling ELISA kit (Active Motif, Tokyo, Japan), according to the manufacturer's protocol. The in vitro angiogenesis was assessed as the formation of capillary-like structures of HUVECs co-cultured with human diploid fibroblasts as described previously (34). The experimental procedure was carried out according to the instructions provided with the angiogenesis kit (Kurabo). The computer-assisted quantitation of tubule formation was performed as in the in vivo assay.

Statistical analysis. For the assessment of the statistical significance of inter-group differences in the quantitative data, Bonferroni's multiple comparison test was performed after one-way ANOVA. This was followed by Bartlett's test to determine the homology of variance.

\section{Results}

General findings in the CSAA- and CDAA-treated rats. The data for the effective numbers of rats, final body and relative liver weights in all experimental groups are shown in Table I. The SAB treatment did not cause any alteration in several indices, such as the ALT level and Alb. Neither ascites nor other organ abnormalities were observed at the end of the experiment in any of the groups. In the CDAA model, the final body and relative liver weights of the CDAA-treated rats (G2, and 3) were less than those of the CSAA-treated rats (G1), as stated in previous reports (35). The relative liver weights in $\mathrm{G} 2$ and $\mathrm{G} 3$ were greater than those in G1.

Effects of $S A B$ on the liver fibrosis development. We first examined the effects of clinically comparable doses of SAB on the development of liver fibrosis. As shown in Fig. 1, SAB-treatment (G3) showed a significant inhibitory effect on liver fibrosis development compared to the control group (G2) $(\mathrm{P}<0.01)$. Semi-quantitative analysis revealed that the fibrosis area in G3 was almost half that in G2. We then carried out an immunohistochemical analysis of $\alpha$-SMA to examine the
A

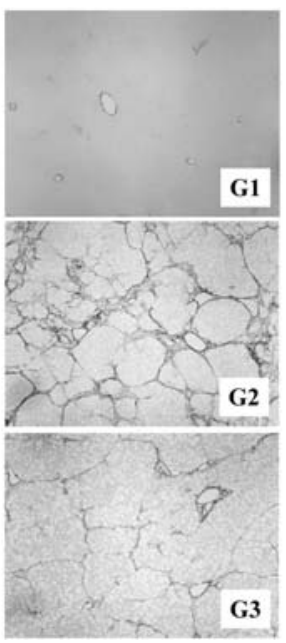

B

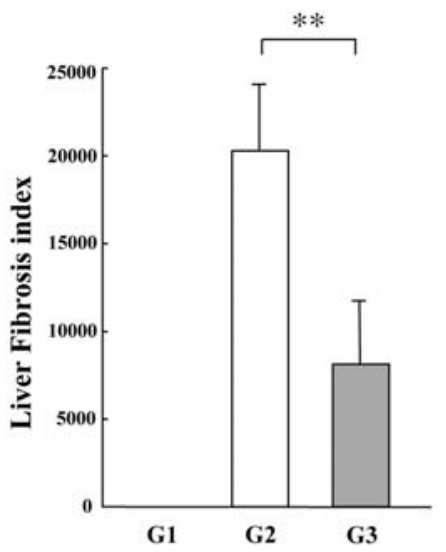

Figure 1. Effects of the SAB on liver fibrosis development. (A) Representative features of microphotographs in the liver sections from the CDAA-treated rats. No fibrosis development could be observed in the CSAA-treated control rats. The CDAA-treatment showed an extensive fibrosis development along with fatty accumulation, and SAB-treatment showed a significant inhibitory effect on liver fibrosis development at a clinically comparable low dose $(100 \mathrm{mg} / \mathrm{kg})$. (B) Semi-quantitative analysis revealed that the fibrosis area in G3 was almost half that in G2. The data represent the mean $\pm \mathrm{SD}$ (bars, $\mathrm{n}=10$ ). ${ }^{* *}$ Indicates a statistically significant difference between the indicated experimental groups $(\mathrm{P}<0.01)$. G1, CSAAtreated control group; G2 and G3, CDAA-treated and CDAA with SABtreated group, respectively.

effect of SAB on HSC activation during liver fibrosis development. The activated HSC, which express $\alpha$-SMA, were drastically reduced in the liver of the SAB-treated groups. This was similar to the effect on fibrosis development (Fig. 2) $(\mathrm{P}<0.01)$. We also examined TGF- $\beta$, which is mainly produced by the activated HSC. Compared to the rats that received a CDAA diet, the ones that received $\mathrm{SAB}$ showed a decrease in TGF- $\beta 1$ in the liver $(\mathrm{P}<0.01)$ (Fig. 4A). The inhibitory effect of $\mathrm{SAB}$ on the TGF- $\beta 1$ expression was almost in parallel with the inhibition of liver fibrosis.

Our in vitro study revealed that Ald at $10^{-8} \mathrm{M}$ significantly stimulated the activated HSC proliferation compared to the untreated control, and that the Ald-induced activated HSC proliferation was suppressed by $\mathrm{SAB}$ in a dose-dependent manner (Fig. 5A).

Effects of SAB on the development of pre-neoplastic lesions. We then examined the effect of SAB on the GST-P-preneoplastic lesions in conjunction with neovascularization. As shown in Fig. 3A, similar to the effect on liver fibrosis inhibition, SAB-treatment (G3) exerted a significant inhibitory effect on the GST-P-positive pre-neoplastic lesion development compared to the control group $(\mathrm{P}<0.01)$. In the CSAAtreated rats, no GST-P-positive lesions were found. In order to determine whether the inhibitory effect of SAB on the preneoplastic lesion development was accompanied by the suppression of neovascularization, we examined the expression level of $\mathrm{CD} 31$. The treatment of $\mathrm{SAB}$ significantly attenuated the CD31-positive vessels in the liver along with the inhibition of GST-P-induced pre-neoplastic lesions (Fig. 3B). In order to elucidate whether the suppressive effects 
A

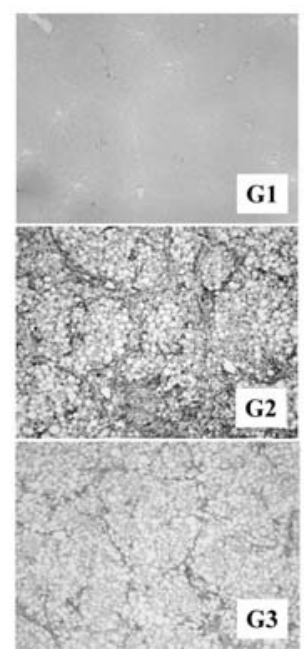

B

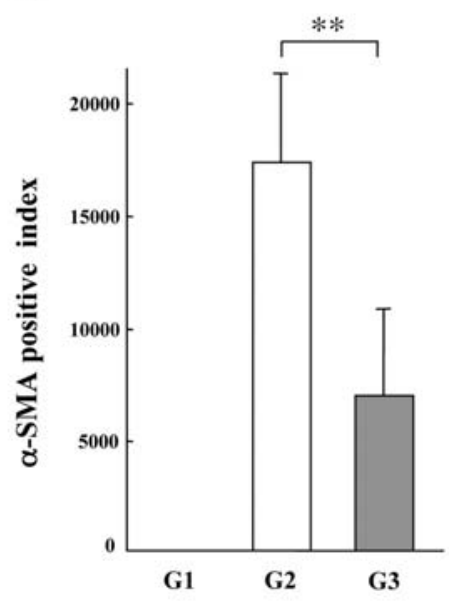

Figure 2. Effects of SAB on the activated HSC in liver fibrosis development. (A) Immunohistochemical analysis of the $\alpha$-SMA expression in the liver from CSAA-diet rats, CDAA-diet rats and SAB-treated-CDAA-diet rats. (B) The $\alpha$-SMA-positive cells were measured with an image analyzer system as described in the Materials and methods. Treatment with SAB showed a marked inhibitory effect on the $\alpha$-SMA expression in the liver as compared to the CDAA-diet group. No significant $\alpha$-SMA expression was observed in G1. The data represent the mean \pm SD (bars, $n=10$ ). ${ }^{* *}$ Indicates a statistically significant difference between the indicated experimental groups $(\mathrm{P}<0.01)$. G1, CSAA-treated control group; G2 and G3, CDAA-treated and CDAA with SAB-treated group, respectively.

A

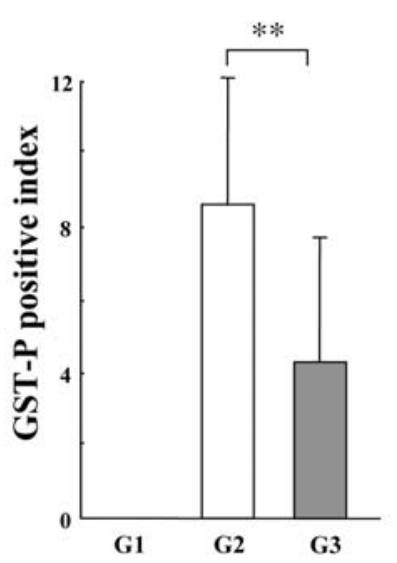

B

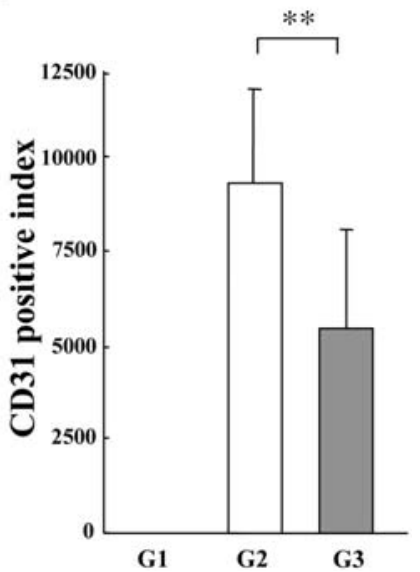

Figure 3. Effects of SAB on the pre-neoplastic lesions and neovascularization. (A) SAB-treatment exerted a significant inhibitory effect on the GST-Ppositive pre-neoplastic lesion development compared to the CDAA-treated group. In the CSAA-treated rats, no GST-P-positive lesions were found. (B) Treatment with SAB significantly attenuated the CD31-positive vessels in the liver and this was almost in conjunction with the inhibition of GST-Ppre-neoplastic lesions. The data represent the mean \pm SD (bars, $n=10$ ). ${ }^{* *}$ Indicates statistically significant difference between the indicated experimental groups $(\mathrm{P}<0.01)$. G1, CSAA-treated control group; G2 and G3, CDAA-treated and CDAA with SAB-treated group, respectively.

of SAB on neovascularization were accompanied with the inhibition of VEGF, we examined the VEGF expression in the liver. As shown in Fig. 4B, the VEGF expression in the liver was suppressed by the treatment with $\mathrm{SAB}(\mathrm{P}<0.01)$. We then performed a set of in vitro experiments. Ald at $10^{-8} \mathrm{M}$ significantly stimulated the in vitro EC proliferation compared

A

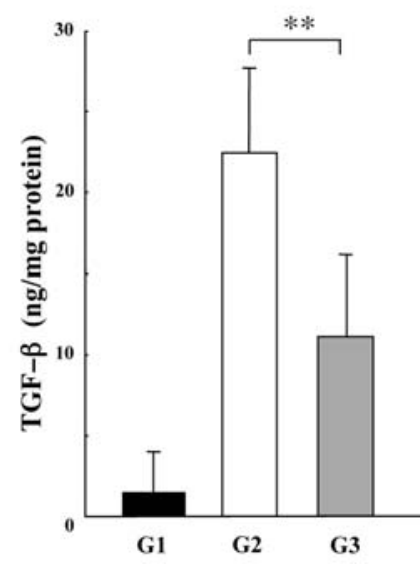

B

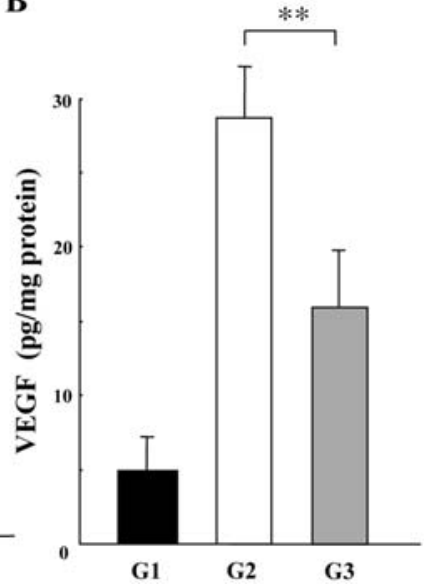

Figure 4. Effects of SAB on the hepatic TGF- $B$ and VEGF protein expression. The TGF- $B$ (A) and VEGF (B) protein levels were measured by ELISA as described in the Materials and methods. Treatment with SAB showed a significant inhibitory effect on the TGF- $\beta$ and VEGF protein expression in the liver compared to the CDAA-diet group. The data represent the mean $\pm \mathrm{SD}$ (bars, $\mathrm{n}=10$ ). ${ }^{* *}$ Indicates a statistically significant difference between the indicated experimental groups $(\mathrm{P}<0.01)$. G1, CSAAtreated control group; G2 and G3, CDAA-treated and CDAA with SABtreated group, respectively.

A

B
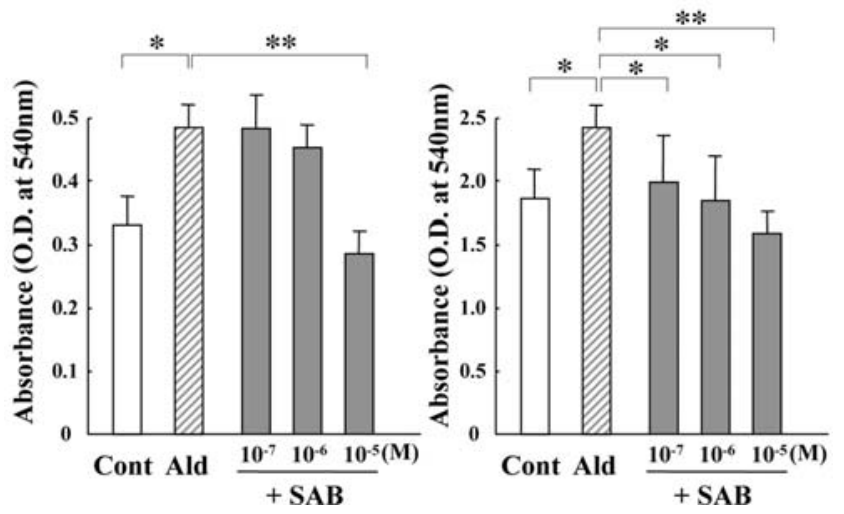

Figure 5. Effects of Ald and SAB on the activated HSC and EC proliferation in vitro. The cells were incubated in the presence of Ald (Ald) and SAB for $24 \mathrm{~h}$, and the proliferation was measured by MTT assay as described in Materials and methods. (A) The Ald $\left(10^{-8} \mathrm{M}\right)$-induced activated HSC proliferation was suppressed by the treatment with SAB (from $10^{-7}$ to $10^{-5} \mathrm{M}$ ) in a dose-dependent manner. (B) The Ald $\left(10^{-8} \mathrm{M}\right)$-induced EC proliferation was suppressed by the treatment with SAB (from $10^{-7}$ to $10^{-5} \mathrm{M}$ ) in a dosedependent manner. Each bar represents the mean \pm SD $(n=5) .{ }^{* * * *}$ Indicate statistically significant differences between the indicated experimental groups $(\mathrm{P}<0.05$ and $<0.01$, respectively).

to the untreated control, and the Ald-induced EC proliferation was suppressed by SAB in a dose-dependent manner (Fig. 5B). We also investigated the in vitro EC tubule formation in the presence or absence of Ald and SAB. Similar to the proliferation findings, Ald significantly stimulated the EC tubule formation compared to the control. SAB significantly inhibited the Ald-induced EC tubule formation (Fig. 6A). We measured the total ERK1/2 (t-ERK) expression and the phosphorylated ERK1/2 (p-ERK) expression in EC. 
A

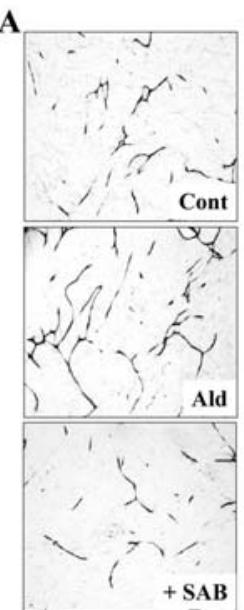

B

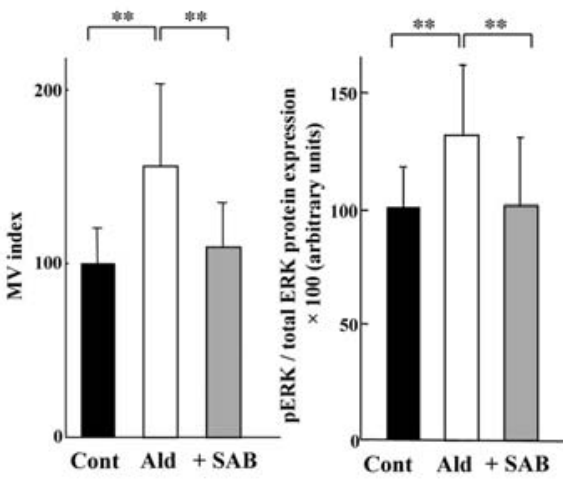

Figure 6. Effects of SAB on the EC tubular formation and on ERK1/2 in EC. (A) Representative features and index of the in vitro EC tubular formation with the Ald and SAB treatment. Cont, untreated control group; Ald, Ald (10-8 M)-treated group; SAB, Ald $\left(10^{-8} \mathrm{M}\right)$ - and SAB $\left(10^{-5} \mathrm{M}\right)$ treated group. Treatment with Ald increased the EC tubule formation compared to the untreated control group, and SAB inhibited the Ald-induced tubule formation. (B) ERK 1/2 phosphorylation in EC with Ald and SAB treatment. The phosphorylated ERK $1 / 2$ significantly increased in the Aldtreated EC, and the Ald-induced ERK 1/2 phosphorylation was suppressed by treatment with SAB. Similar to the EC proliferation, SAB suppressed the ERK 1/2 phosphorylation. Cont, untreated control group; Ald, Ald $\left(10^{-8} \mathrm{M}\right)$ treated group; SAB, Ald $\left(10^{-8} \mathrm{M}\right)$ - and SAB $\left(10^{-5} \mathrm{M}\right)$-treated group. The results are expressed as the ratio of the phosphorylated ERK 1/2 (pERK) to the total ERK $1 / 2$ in EC. The data represent the mean \pm SD (bars, $n=8$ ). ** Indicates a statistically significant difference between the indicated experimental groups $(\mathrm{P}<0.01)$.

As shown in Fig. 6B, Ald significantly increased p-ERK whereas no marked change could be observed in t-ERK. SAB significantly suppressed the Ald-induced p-ERK and the ratio of $\mathrm{p}-\mathrm{ERK} / \mathrm{t}-\mathrm{ERK}$.

\section{Discussion}

Ald is secreted by the cells of the zona glomerulosa upon stimulation with Ang-II, and it regulates blood volume homeostasis. Besides this hormonal effect, Ald can be synthesized in the extra-adrenal tissue, e.g., heart and blood vessels (36-38). Ald is now recognized as a multifunctional protein. Recent studies have shown that Ald participates in endothelial dysfunction, vascular fibrosis, and in the inflammation of the vasculature. Although its deleterious pro-fibrogenic effects on the cardiovascular system are well known, little information is available on the Ald effects on liver fibrogenesis (38-40). In the current study, we revealed that Ald plays an important role in liver fibrosis development, and that SAB markedly attenuated fibrogenesis along with the suppression of the activated HSC. The hepatic level of TGF- $\beta$, which is mainly produced in the activated HSC, was also suppressed by treatment with $\mathrm{SAB}$ at a similar magnitude. These results confirmed that the anti-fibrotic effect of SAB was achieved by the suppression of HSC activation. Previous reports have shown that anti-Ald agents, such as spironolactone and canrenone, could suppress experimental liver fibrosis (40-42). These anti-Ald drugs are commonly used as diuretics in the treatment of cirrhotic ascites, either alone or in association with loop diuretics. However, their use is associated with progestational and anti-androgenic side effects due to their promiscuous binding to other steroid receptors. These are most commonly manifested as gynecomastia, an abnormal menstrual cycle, and impotence, limiting their use by physicians in chronic liver disease. On the contrary, SAB is a selective MR antagonist with minimal binding to other steroid receptors such as progesterone and androgen, and its selectivity is 100 times more than that of spironolactone. Considering that long-term administration is required for anti-fibrotic treatment, and taking into account the previous reports on spironolactone, our study on SAB proves that it is a more likely candidate for future clinical application.

In addition to liver fibrosis development, we observed the inhibitory effects of SAB on pre-neoplastic lesions along with the suppression of neovascularization and VEGF in the liver. Regarding tumor growth, recent studies have revealed that angiogenesis begins at a very early stage when the neoplastic cells contain only a few hundreds cells $(43,44)$. We have previously reported that the hepatic neovascularization increased stepwise during hepatocarcinogenesis $(19,20,45)$. As such, we found neovascularization mainly in the GST-Ppositive lesions compared to the adjacent areas. It has been suggested that the anti-angiogenic agents exert a much more potent inhibitory effect on the size rather than on the number of pre-neoplastic lesions (5). We have previously reported that SAB exerted a significant inhibitory effect on the VEGFmediated neovascularization in HCC (24). This could be the reason why $\mathrm{SAB}$ exerted a marked inhibitory effect on the size of the pre-neoplastic lesions, but not on the number of lesions. The direct interaction between the SAB treatment and angiogenesis during hepatocarcinogenesis is currently being evaluated at our laboratory.

Although previous studies conducted to determine the molecular processes associated with fibrosis and angiogenesis were performed independently, recent studies have revealed that both biological phenomena emerged synergistically $(46,47)$. We, as well as others, reported that neovascularization significantly increased during liver fibrosis development (48-51). In the current study, we showed that, as in EC, Ald acted on the activated HSC. Accordingly, it is possible that the augmentation of Ald-mediated neovascularization could also play a certain role in the development of liver fibrosis.

The CDAA diet induces histological changes similar to those of the human NASH, and therefore this model is widely used as a NASH model $(10,25,51)$. We also used this model to elucidate the effect of $\mathrm{SAB}$ on the progression of $\mathrm{SAB}$, as was done for ARB in a previous study (10). However, a downside of the CDAA model is that it does not exhibit several common features of NASH, such as insulin resistance and diabetes mellitus. In order to examine the precise pharmacological action of any drug, it is important to examine its therapeutic effect under the condition of insulin resistance. We previously investigated the effect of ARB on CDAA-induced liver fibrosis development in Otsuka LongEvans Tokushima fatty (OLETF) rats, which commonly have obesity, diabetes mellitus, and insulin resistance. The 
therapeutic effect of SAB in the CDAA-induced OLETF rats remains to be elucidated.

In summary, we showed here that Ald plays an important role in the progression of NASH. The treatment with SAB significantly inhibited liver fibrogenesis and carcinogenesis, at least partly, via the suppression of activated HSC and neovascularization, respectively. As this agent is already widely used in clinical practice with great safety, it could represent a potential new therapeutic strategy against the progression of NASH in the future.

\section{References}

1. Angulo P: Nonalcoholic fatty liver disease. N Engl J Med 346: 1221-1231, 2002.

2. Page JM and Harrison SA: Nash and HCC. Clin Liver Dis13: 631-647, 2009

3. Kawada N, Imanaka K, Kawaguchi T, et al: Hepatocellular carcinoma arising from non-cirrhotic nonalcoholic steatohepatitis. J Gastroenterol 44: 1190-1194, 2009.

4. Yun JW, Cho YK, Park JH, et al: Abnormal glucose tolerance in young male patients with nonalcoholic fatty liver disease. Liver Int 29: 525-529, 2009.

5. Yoshiji H, Yoshii J, Ikenaka Y, et al: Inhibition of reninangiotensin system attenuates liver enzyme-altered preneoplastic lesions and fibrosis development in rats. J Hepatol 37: 22-30, 2002.

6. Yoshiji H, Kuriyama S, Noguchi R and Fukui H: Angiotensin-i converting enzyme inhibitors as potential anti-angiogenic agents for cancer therapy. Curr Cancer Drug Targets 4: 555-567, 2004.

7. Yoshiji H, Kuriyama S and Fukui H: Angiotensin-i-converting enzyme inhibitors may be an alternative anti-angiogenic strategy in the treatment of liver fibrosis and hepatocellular carcinoma. Possible role of vascular endothelial growth factor. Tumour Biol 23: 348-356, 2002.

8. Helmy A, Jalan R, Newby DE, Hayes PC and Webb DJ: Role of angiotensin ii in regulation of basal and sympathetically stimulated vascular tone in early and advanced cirrhosis. Gastroenterology 118: 565-572, 2000.

9. Asbert M, Jimenez W, Gaya J, et al: Assessment of the reninangiotensin system in cirrhotic patients. Comparison between plasma renin activity and direct measurement of immunoreactive renin. J Hepatol 15: 179-183, 1992.

10. Yoshiji H, Noguchi R, Ikenaka Y, et al: Losartan, an angiotensin-ii type 1 receptor blocker, attenuates the liver fibrosis development of non-alcoholic steatohepatitis in the rat. BMC Res Notes 2: 70, 2009

11. Yokohama S, Yoneda M, Haneda M, et al: Therapeutic efficacy of an angiotensin II receptor antagonist in patients with nonalcoholic steatohepatitis. Hepatology 40: 1222-1225, 2004.

12. Tox U and Steffen HM: Impact of inhibitors of the reninangiotensin-aldosterone system on liver fibrosis and portal hypertension. Curr Med Chem 13: 3649-3661, 2006.

13. Yoshiji H, Kuriyama S, Noguchi R, et al: Amelioration of carcinogenesis and tumor growth in the rat liver by combination of vitamin K2 and angiotensin-converting enzyme inhibitor via anti-angiogenic activities. Oncol Rep 15: 155-159, 2006.

14. Yoshiji H, Kuriyama S, Yoshii J, et al: Vascular endothelial growth factor tightly regulates in vivo development of murine hepatocellular carcinoma cells. Hepatology 28: 1489-1496, 1998 .

15. Noguchi R, Yoshiji H, Kuriyama S, et al: Combination of interferon-beta and the angiotensin-converting enzyme inhibitor perindopril, attenuates murine hepatocellular carcinoma development and angiogenesis. Clin Cancer Res 9: 6038-6045, 2003.

16. Kerbel RS: Tumor angiogenesis: Past, present and the near future. Carcinogenesis 21: 505-515, 2000.

17. Yamaguchi R, Yano H, Iemura A, Ogasawara S, Haramaki M and Kojiro M: Expression of vascular endothelial growth factor in human hepatocellular carcinoma. Hepatology 28: 68-77, 1998.

18. Poon RT, Ng IO, Lau C, et al: Serum vascular endothelial growth factor predicts venous invasion in hepatocellular carcinoma: A prospective study. Ann Surg 233: 227-235, 2001.
19. Yoshiji H, Kuriyama S, Yoshii J, et al: Halting the interaction between vascular endothelial growth factor and its receptors attenuates liver carcinogenesis in mice. Hepatology 39: 15171524,2004

20. Yoshiji H, Kuriyama S, Yoshii J, et al: Involvement of the vascular endothelial growth factor receptor-1 in murine hepatocellular carcinoma development. J Hepatol 41: 97-103, 2004.

21. Romanque P, Piguet AC and Dufour JF: Targeting vessels to treat hepatocellular carcinoma. Clin Sci (Lond) 114: 467-477, 2008.

22. Llovet JM, Ricci S, Mazzaferro V, et al: Sorafenib in advanced hepatocellular carcinoma. N Engl J Med 359: 378-390, 2008.

23. Berenson A: A cancer drug shows promise, at a price that many can't pay. New York Times, February 15, 2006.

24. Kaji K, Yoshiji H, Kitade M, et al: Selective aldosterone blocker, eplerenone, attenuates hepatocellular carcinoma growth and angiogenesis in mice. Hepatol Res, April 14, 2010 (Epub ahead of print).

25. Tsujimoto T, Kawaratani H, Kitazawa T, et al: Decreased phagocytic activity of kupffer cells in a rat nonalcoholic steatohepatitis model. World J Gastroenterol 14: 6036-6043, 2008.

26. Delyani JA, Rocha R, Cook CS, et al: Eplerenone: A selective aldosterone receptor antagonist (SARA). Cardiovasc Drug Rev 19: 185-200, 2001.

27. Yoshiji H, Kuriyama S, Yoshii J, et al: The copper-chelating agent, trientine, attenuates liver enzyme-altered preneoplastic lesions in rats by angiogenesis suppression. Oncol Rep 10: 1369-1373, 2003.

28. Noguchi R, Yoshiji H, Ikenaka Y, et al: Synergistic inhibitory effect of gemcitabine and angiotensin type-1 receptor blocker, losartan, on murine pancreatic tumor growth via anti-angiogenic activities. Oncol Rep 22: 355-360, 2009.

29. Yoshiji H, Kuriyama S, Kawata M, et al: The angiotensin-iconverting enzyme inhibitor perindopril suppresses tumor growth and angiogenesis: Possible role of the vascular endothelial growth factor. Clin Cancer Res 7: 1073-1078, 2001.

30. Yoshiji H, Kuriyama S, Yoshii J, et al: Angiotensin-ii type 1 receptor interaction is a major regulator for liver fibrosis development in rats. Hepatology 34: 745-750, 2001.

31. Weiskirchen R and Gressner AM: Isolation and culture of hepatic stellate cells. Methods Mol Med 117: 99-113, 2005.

32. Rockey DC, Boyles JK, Gabbiani G and Friedman SL: Rat hepatic lipocytes express smooth muscle actin upon activation in vivo and in culture. J Submicrosc Cytol Pathol 24: 193-203, 1992.

33. Yoshiji H, Kuriyama S, Yoshii J, et al: Synergistic effect of basic fibroblast growth factor and vascular endothelial growth factor in murine hepatocellular carcinoma. Hepatology 35: 834-842, 2002.

34. Yoshiji H, Kuriyama S, Noguchi R, et al: Combination of vitamin $\mathrm{K} 2$ and the angiotensin-converting enzyme inhibitor, perindopril, attenuates the liver enzyme-altered preneoplastic lesions in rats via angiogenesis suppression. J Hepatol 42: 687-693, 2005.

35. Nakae D, Yoshiji H, Mizumoto Y, et al: High incidence of hepatocellular carcinomas induced by a choline deficient Lamino acid defined diet in rats. Cancer Res 52: 5042-5045, 1992.

36. Takeda Y, Miyamori I, Yoneda T, et al: Synthesis of corticosterone in the vascular wall. Endocrinology 135: 2283-2286, 1994.

37. Delcayre C and Swynghedauw B: Molecular mechanisms of myocardial remodeling. The role of aldosterone. J Mol Cell Cardiol 34: 1577-1584, 2002.

38. Yang X, Li X, Wu P, Meng Y, Li S and Lai W: Cyp11b2 expression in rat liver and the effect of spironolactone on hepatic fibrogenesis. Horm Res 53: 288-293, 2000.

39. Li X, Meng Y, Wu P, Zhang Z and Yang X: Angiotensin II and aldosterone stimulating NF-kappaB and AP-1 activation in hepatic fibrosis of rat. Regul Pept 138: 15-25, 2007.

40. Caligiuri A, De Franco RM, Romanelli RG, et al: Antifibrogenic effects of canrenone, an antialdosteronic drug, on human hepatic stellate cells. Gastroenterology 124: 504-520, 2003.

41. Fujisawa G, Muto S, Okada K, Kusano E and Ishibashi S: Mineralocorticoid receptor antagonist spironolactone prevents pig serum-induced hepatic fibrosis in rats. Transl Res 148: 149-156, 2006

42. Rombouts K, Niki T, Wielant A, et al: Effect of aldosterone on collagen steady state levels in primary and subcultured rat hepatic stellate cells. J Hepatol 34: 230-238, 2001. 
43. Li CY, Shan S, Huang Q, et al: Initial stages of tumor cellinduced angiogenesis: Evaluation via skin window chambers in rodent models. J Natl Cancer Inst 92: 143-147, 2000.

44. Frachon S, Gouysse G, Dumortier J, et al: Endothelial cell marker expression in dysplastic lesions of the liver: An immunohistochemical study. J Hepatol 34: 850-857, 2001.

45. Yoshiji H, Kuriyama S, Hicklin DJ, et al: KDR/Flk-1 is a major regulator of vascular endothelial growth factor-induced tumor development and angiogenesis in murine hepatocellular carcinoma cells. Hepatology 30: 1179-1186, 1999.

46. Paternostro C, David E, Novo E and Parola M: Hypoxia, angiogenesis and liver fibrogenesis in the progression of chronic liver diseases. World J Gastroenterol 16: 281-288, 2010 .
47. Valfre di Bonzo L, Novo E, Cannito S, et al: Angiogenesis and liver fibrogenesis. Histol Histopathol 24: 1323-1341, 2009.

48. Yoshiji H, Kuriyama S, Yoshii J, et al: Vascular endothelial growth factor and receptor interaction is a prerequisite for murine hepatic fibrogenesis. Gut 52: 1347-1354, 2003.

49. Kitade M, Yoshiji H, Kojima H, et al: Leptin-mediated neovascularization is a prerequisite for progression of nonalcoholic steatohepatitis in rats. Hepatology 44: 983-991, 2006.

50. Amarapurkar AD, Amarapurkar DN, Vibhav S and Patel ND: Angiogenesis in chronic liver disease. Ann Hepatol 6: 170-173, 2007.

51. Ueno T, Nakamura T, Torimura T and Sata M: Angiogenic cell therapy for hepatic fibrosis. Med Mol Morphol 39: 16-21, 2006. 